\section{Use of Belimumab throughout 2 Consecutive Pregnancies in a Patient with Systemic Lupus Erythematosus}

To the Editor:

Effects of belimumab on pregnant patients with systemic lupus erythematosus (SLE) are unknown. To our knowledge, we described the first case of a patient with active SLE who was treated with belimumab throughout her pregnancy ${ }^{1}$. The patient became pregnant again and we describe its successful outcome here.

Ethical board approval for case reports is not required per institution policy. Informed consent was obtained from the patient.

A 41-year-old woman with difficult-to-control SLE (antinuclear antibody-positive, SSA, dsDNA, antigranulocyte antibody-positive, and lupus nephritis) reported to be pregnant again while receiving belimumab. Belimumab was used successfully during her first pregnancy because of contraindications or side effects to azathioprine, mycophenolate, rituximab, and cyclophosphamide (Table 1). Her second conception resulted in a miscarriage, which was because of aneuploidy and was not thought to be because of SLE. Her SLE was in remission for 18 months before her third conception (Table 1). She maintained treatment with belimumab $10 \mathrm{mg} / \mathrm{kg}$, hydroxychloroquine $400 \mathrm{mg}$, prednisone $5 \mathrm{mg}$, and low molecular weight heparin. She had intensive monitoring with monthly ultrasound, noninvasive prenatal testing, and fetal echocardiography. Serial testing did not reveal any developmental abnormalities, heart blockage, or aneuploidy. Her SLE was in clinical remission during her pregnancy (Table 1). She had a history of longstanding leukopenia that was monitored and had no infections. Belimumab was successful in controlling all her SLE manifestations apart from her leukopenia. Her belimumab infusion was continued until 33 weeks of gestation. Her non-stress test done at 37 weeks showed a normal fetal heart rate with moderate variability and accelerations. The patient had a planned Cesarean delivery at 39 weeks. A healthy baby boy was born: 19 inches long, head circumference of 14 inches, and weight 7 pounds, 6.9 ounces. He had normal Apgar scores at birth. The baby did not have any evidence of Ebstein anomaly as was noted to be present in the patient's first baby. He did not have any congenital heart blocks despite the positive maternal SSA serology. He did have an extrarenal pelvis and was followed closely by pediatric urology. Belimumab was restarted 3 weeks after the delivery. The patient and baby continue to do well a year later.

Effective management of SLE before conception and during pregnancy has been shown to improve outcomes ${ }^{2}$. A very limited number of medications can be used in SLE without having a potential harmful effect on the baby. Belimumab was successful in achieving good SLE control during both pregnancies in our patient. Her second baby did have an extrarenal pelvis, which is considered a normal anatomic variant ${ }^{3}$. Our patient did have leukopenia prior to the initiation of belimumab and she continued to have grade 3 or grade 4 leukopenia. Leukopenia has been reported in the clinical trials on belimumab $b^{4,5,6}$, and the cause of our patient's leukopenia is likely a combination of SLE activity and belimumab use. The role of her antigranulocytic antibodies in the development of leukopenia cannot be ruled out.

To our knowledge, there are no human clinical studies available evaluating the use of belimumab in pregnant women. Belimumab crosses the placenta in pregnant monkeys in concentrations that result in reversible pharmacologic activity in fetuses and newborn monkeys ${ }^{7}$. Pregnancy was an exclusion criterion in all belimumab trials. The pooled data from placebo-controlled belimumab studies reported 6 pregnancies in the placebo arm, and there were no live births ${ }^{4}$. Hydroxychloroquine use was $63 \%$, corticosteroid use was $81 \%$, and other immunosuppressant use was $48 \%$ in the belimumab group ${ }^{4}$.

The Belimumab Pregnancy Registry (BPR $)^{8}$ aims to evaluate pregnancy and infant outcomes in women with SLE exposed to commercially supplied belimumab within the 4 months prior to and/or during pregnancy. As of March 2016, there were 21 participants with confirmed outcomes; 19 were exposed to belimumab within 4 months prior to conception, 1 patient was exposed in the first trimester, and the timing of exposure in the elective termination was not evaluated. There were 17 participants who had live births ( 1 twin pregnancy; 18 total live births), 1 had elective termination of pregnancy (no apparent congenital anomaly reported), and 3 had spontaneous miscarriages. Four of 18 live births were reported to have congenital anomalies ${ }^{8,9}$. Cumulative data from clinical trials, spontaneous reports, BPR, and postmarketing surveillance have reported a total of 254 pregnancies (Table 2) ${ }^{9}$. Congenital abnormalities reported in live infants of patients exposed to belimumab included Dandy Walker syndrome, bilateral enlarged kidneys, pulmonic stenosis, mild Ebstein anomaly (our patient's first child), unbalanced translocation between chromosomes 11 and 13, bilateral club foot, and ventricular septal defect. The data from the BPR, though limited, demonstrate that patients receiving belimumab can have successful pregnancy outcomes. There is still insufficient evidence to prove the safety of belimumab during pregnancy. Additional data from the BPR and postmarketing surveillance over the coming years will be of value in answering this.

Table 1. Laboratory data from first and second successful pregnancy.

\begin{tabular}{|c|c|c|c|c|c|c|c|c|}
\hline Time & $\mathrm{Hb}, \mathrm{mg} / \mathrm{dl}$ & $\begin{array}{l}\text { WBC, } \\
\text { per } \mathrm{cm}^{2}\end{array}$ & $\begin{array}{c}\text { Platelet } \\
\text { Count, } \mathrm{k} / \mathrm{cm}^{2}\end{array}$ & $\begin{array}{c}\text { Serum } \\
\text { Creatinine, } \mathrm{mg} / \mathrm{dl}\end{array}$ & $\begin{array}{l}\text { UPCR, } \\
\mathrm{mg} / \mathrm{mg}\end{array}$ & $\begin{array}{c}\mathrm{C} 3,88-201 \\
\mathrm{mg} / \mathrm{dl}\end{array}$ & $\begin{array}{c}\mathrm{C} 4,16-47 \\
\mathrm{mg} / \mathrm{dl}\end{array}$ & $\begin{array}{l}\text { dsDNA } \\
\text { Titer }\end{array}$ \\
\hline 3 mos before belimumab & 11.9 & 8.2 & 215 & 1.12 & 0.11 & 62 & 8 & $1: 80$ \\
\hline 6 mos after belimumab & 11.8 & 2.6 & 170 & 0.77 & 0.14 & 73 & 22 & - \\
\hline 12 mos after belimumab & 12 & 2.1 & 140 & 0.83 & 0.25 & 59 & 16 & - \\
\hline First trimester, first pregnancy & 11.7 & 1.9 & 161 & 0.59 & 0.14 & 87 & 24 & $1: 40$ \\
\hline Postpartum, first pregnancy & 11.9 & 0.9 & 195 & 0.78 & - & 73 & 22 & $1: 80$ \\
\hline 3 mos before second pregnancy & 12.1 & 1.37 & 274 & 0.79 & 0.12 & 122 & 36 & $1: 80$ \\
\hline First trimester, $\sim 8$ weeks & 13.0 & 1.58 & 246 & 0.78 & - & - & - & - \\
\hline Second trimester, $\sim 20$ weeks & 10.7 & 1.84 & 205 & 0.64 & 0.15 & 140 & 46 & - \\
\hline Third trimester, $\sim 34$ weeks & 12.0 & 1.46 & 175 & 0.73 & 0.16 & - & - & - \\
\hline \multicolumn{9}{|l|}{3 weeks postpartum, first belimumab } \\
\hline
\end{tabular}

Hb: hemoglobin; WBC: white blood cell; UPCR: urine protein creatinine ratio. 
Table 2. Cumulative pregnancy outcomes from clinical trials, spontaneous reports, postmarketing surveillance, and the Belimumab Pregnancy Registry.

\begin{tabular}{lc}
\hline Variables & $\mathrm{n}$ \\
\hline Total pregnancies & 254 \\
Lost to followup & 45 \\
Total pregnancies with known outcomes & 182 \\
Elective termination, no apparent congenital anomaly & 48 \\
Total pregnancies with known outcomes excluding & \\
$\quad$ elective termination & 134 \\
Total live births & 90 \\
Live infant with no apparent congenital anomaly & 82 \\
Live infant with congenital anomaly & 8 \\
\end{tabular}

ANAND KUMTHEKAR, MD, Fellow in Training, Division of Arthritis and Rheumatic Diseases, Oregon Health and Science University (OHSU), Portland, Oregon; ABHIJEET DANVE, MD, Assistant Clinical Professor of Medicine, Division of Rheumatology, Yale University, New Haven, Connecticut; ATUL DEODHAR, MD, Professor of Medicine, Division of Arthritis and Rheumatic Diseases, OHSU, Portland, Oregon, USA. Address correspondence to Dr. A. Kumthekar, Oregon Health and Science University, Division of Arthritis and Rheumatic Diseases (Mail code: OP09), 3181 SW Sam Jackson Park Road, Portland, Oregon 97239, USA.

E-mail: kumthean@ohsu.edu

\section{REFERENCES}

1. Danve A, Perry L, Deodhar A. Use of belimumab throughout pregnancy to treat active systemic lupus erythematosus: a case report. Semin Arthritis Rheum 2014;44:195-7.

2. Buyon JP, Kim MY, Guerra MM, Laskin CA, Petri M, Lockshin $\mathrm{MD}$, et al. Predictors of pregnancy outcome in a prospective, multiethnic cohort of lupus patients. Ann Intern Med 2015; 163:153-63.
3. Lam BC, Wong SN, Yeung CY, Tang MH, Ghosh A. Outcome and management of babies with prenatal ultrasonographic renal abnormalities. Am J Perinatol 1993;10:263-8.

4. Wallace DJ1, Navarra S, Petri MA, Gallacher A, Thomas M, Furie R, Levy RA, et al; BLISS-52 and -76, and LBSL02 Study Groups. Safety profile of belimumab: pooled data from placebo-controlled phase 2 and 3 studies in patients with systemic lupus erythematosus. Lupus 2013;22:144-54.

5. Manzi S, Sánchez-Guerrero J, Merrill JT, Furie R, Gladman D, Navarra SV, et al; BLISS-52 and BLISS-76 Study Groups. Effects of belimumab, a B lymphocyte stimulator-specific inhibitor, on disease activity across multiple organ domains in patients with systemic lupus erythematosus: combined results from two phase III trials. Ann Rheum Dis 2012;71:1833-8.

6. Yamada M, Akita M, Nakagawa T, Takahashi N, Endo A, Yoshida P. Safety, tolerability, pharmacokinetics and pharmacodynamics of belimumab in Japanese patients with mild-to-moderate systemic lupus erythematosus. J Drug Assess 2013;2:40-8.

7. Auyeung-Kim DJ, Devalaraja MN, Migone TS, Cai W, Chellman GJ. Developmental and peri-postnatal study in cynomolgus monkeys with belimumab, a monoclonal antibody directed against B-lymphocyte stimulator. Reprod Toxicol 2009;28:443-55.

8. Bassiri A. The Belimumab Pregnancy Registry. Glakso Smith Kline (GSK) group of companies. March 2016. Data on file.

9. Cumulative pregnancy outcomes for belimumab from clinical trials, spontaneous reports, post-marketing surveillance reports, and the Belimumab Pregnancy Registry (BPR). Glakso Smith Kline (GSK) group of companies. March 2016. Data on file.

J Rheumatol 2017;44:9; doi:10.3899/jrheum.170327 